\title{
Links and risks associated with psoriasis and metabolic syndrome
}

\author{
This article was published in the following Dove Press journal: \\ Psoriasis: Targets and Therapy \\ 2 November 2015 \\ Number of times this article has been viewed
}

\section{Aikaterini I Liakou Christos C Zouboulis \\ Departments of Dermatology, Venereology, Allergology and Immunology, Dessau Medical Centre, Dessau, Germany}

Correspondence: Christos C Zouboulis Departments of Dermatology, Venereology, Allergology and Immunology, Dessau Medical Center, Auenweg 38, 06847 Dessau, Germany Tel +49340 50l 4000

$\mathrm{Fax}+493405014025$ Email christos.zouboulis@klinikumdessau.de
Introduction: Psoriasis has long been considered a systemic inflammatory disease. Lately, it has been strongly associated with obesity, as well as other components of metabolic syndrome, such as dyslipidemia, insulin resistance, and arterial hypertension.

Methods: We reviewed the literature of the last decade by using the keywords: psoriasis, metabolic syndrome, and/or obesity in PubMed and Medline.

Results: Obesity and psoriasis seem to share similar profiles of systemic inflammation. Serum cytokines such as TNF- $\alpha$, CRP, IL-6, and IL-12 are elevated in both disorders. The more severely an individual is affected with psoriasis, the more likely it is to be obese. This makes the disease an important health care issue, which requires the cooperation of dermatologists with other medical specialists.

Discussion: This review attempts to summarize the links and risks that associate psoriasis with obesity, and highlight the concerns and queries for both disorders in the future.

Keywords: psoriasis, obesity, metabolic, syndrome, inflammation

\section{Introduction}

Psoriasis was for years considered to be a purely dermatological condition, which severely affects patients' quality of life but does not impact their overall health status. However, this perception has changed increasingly during the last decades. Psoriasis is nowadays considered to be a disease of increased systemic inflammation and has been strongly associated with systemic diseases and pathological conditions, such as obesity, metabolic syndrome, and cardiovascular disorders. It has been linked to an increased risk of myocardial infarction, especially in the more severely affected, younger patients. Psoriasis has also been associated with components of metabolic syndrome, such as hypertension, dyslipidemia, and insulin resistance.

This is not a pathophysiological paradox, since psoriasis and obesity seem to share the same inflammatory profiles and overexpress similar cytokines. IL- 6 , TNF- $\alpha$, adiponectin, and PAI-1 are primary adipocytokines that are also expressed in patients with psoriasis. They play multiple roles in inflammation, metabolism, and endothelial cell function regulation. Serum TNF- $\alpha$ levels, for example, which are overexpressed in psoriasis patients, increase with increasing body mass index (BMI), induce insulin resistance, and cause endothelial cells to produce adhesion molecules. This link of psoriasis with obesity and metabolic syndrome places psoriasis in a more important position in the health care system (Figure 1). ${ }^{1,2}$ 


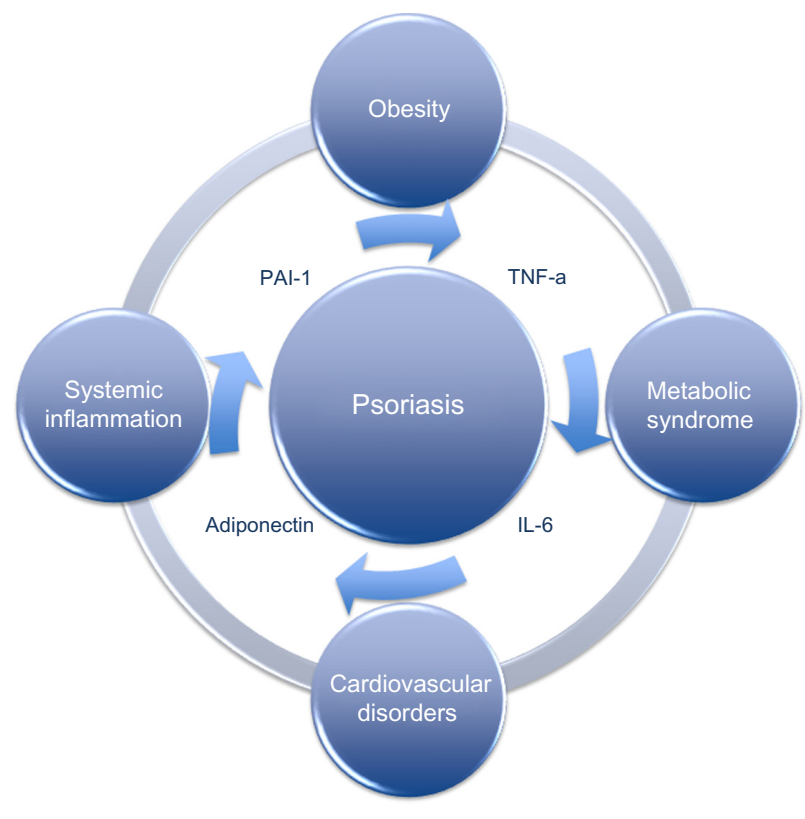

Figure I Psoriasis is a systemic disease of chronic inflammation, which is closely related to other diseases of systemic inflammation, such as obesity, cardiovascular comorbidities, and metabolic syndrome.

\section{Psoriasis, metabolic syndrome, and inflammation}

An association between psoriasis and obesity was first observed in 1995 by Henseler and Christophers. ${ }^{1}$ This association was further supported by clinical studies, especially by those which assessed the efficacy of biologics. In these studies, it was observed that a high percentage of patients with moderate to severe psoriasis were obese. ${ }^{2,3}$ In etanercept trials, $46 \%$ of 3,700 patients had a BMI of $>30 \mathrm{~kg} / \mathrm{m}^{2}$, while the same percentage in the general population in the USA is $31 \%{ }^{4}$

The average BMI of psoriatic patients included in a review of Phase II and III clinical studies on biologics (in more than 10,000 patients) was $30.6 \mathrm{~kg} / \mathrm{m}^{2} .{ }^{1}$ Naldi et al directly related the risk of psoriasis with elevated BMI. ${ }^{5}$ In a study by Krueger et al, a large percentage of patients with psoriasis had an average BMI that significantly exceeded that of the normal population. Moreover, obese patients were more likely to have severe psoriasis (more than $20 \%$ of body surface area). ${ }^{2}$ A study in the UK came to the same conclusion, which not only underlined the fact that psoriasis patients are more likely to be obese than controls, but also that obesity is more prevalent in patients with severe psoriasis. ${ }^{6}$ It is also interesting that a study from Utah, USA, implied that obesity usually follows - not precedes psoriasis, suggesting that psoriatic inflammation helps to induce or worsen obesity. ${ }^{7}$
Intra-abdominal obesity is directly associated with the metabolic syndrome, a condition of persistent systemic inflammation which is diagnosed when at least three of the following five conditions are present: 1) fasting glucose $100 \mathrm{mg} / \mathrm{dL}$ or greater (or receiving drug therapy for hyperglycemia); 2) blood pressure $130 / 85 \mathrm{mmHg}$ or higher (or receiving drug therapy for hypertension); 3) triglycerides $150 \mathrm{mg} / \mathrm{dL}$ or higher (or receiving drug therapy for hypertriglyceridemia); 4) high density lipoprotein cholesterol less than $40 \mathrm{mg} / \mathrm{dL}$ in men or less than $50 \mathrm{mg} / \mathrm{dL}$ in women (or receiving drug therapy to increase high density lipoprotein cholesterol); and 5) waist circumference $102 \mathrm{~cm}$ or greater in men or $88 \mathrm{~cm}$ or greater in women; in Asian Americans, $90 \mathrm{~cm}$ or greater in men or $80 \mathrm{~cm}$ or greater in women (Table 1). This syndrome affects approximately $25 \%$ of the US, $10 \%$ of the French, and $20 \%$ of the Australian populations. ${ }^{8}$ Additionally, elevations in BMI and waist circumference are strongly correlated with increased risk for coronary heart disease. ${ }^{4}$ Many studies have shown that individual features of the metabolic syndrome are more often present in patients with psoriasis. ${ }^{9} 10$

Metabolic syndrome is related to increased levels of proinflammatory cytokines and therefore to systemic inflammation. Intra-abdominal fat seems to be associated with elevated TNF- $\alpha$, IL-6, and PAI-1 levels. TNF- $\alpha$ induces the endothelial cells to produce adhesion molecules and subsequently provoke the adherence of monocytes. TNF- $\alpha$ increases with increasing BMI, and induces insulin resistance, partially also by leading to an elevation in free fatty acids. This is how TNF- $\alpha$ contributes to the early stages of atherosclerosis. IL-6 may also contribute to the pathogenesis of atherosclerosis likewise by inducing insulin resistance, increase of endothelial adhesion molecules, and augmentation of the procoagulant effects on platelets. Finally, TNF- $\alpha$ also increases the levels of PAI-1, which in turn suppresses the activity of tPA, an anticlotting factor. Impaired fibrinolysis and uninhibited clotting occur as a result of the increased levels of PAI-1.11,12

Table I The metabolic syndrome and its components

\begin{tabular}{lll}
\hline Metabolic syndrome & \\
\hline Components & Range of values & $\begin{array}{l}\text { Or receiving drug } \\
\text { therapy }\end{array}$ \\
\hline Fasting glucose & $\geq 100 \mathrm{mg} / \mathrm{dL}$ & For hyperglycemia \\
Blood pressure & $\geq 130 / 85 \mathrm{mmHg}$ & For hypertension \\
Triglycerides & $\geq 150 \mathrm{mg} / \mathrm{dL}$ & For hypertriglyceridemia \\
HDL cholesterol & $<40 \mathrm{mg} / \mathrm{dL}$ (men) & To increase HDL \\
& or $<50 \mathrm{mg} / \mathrm{dL}$ (women) & cholesterol \\
Waist & $\geq 102 \mathrm{~cm}$ (men) & \\
circumference & or $\geq 88 \mathrm{~cm}$ (women) & \\
\hline
\end{tabular}

Abbreviation: HDL, high density lipoprotein. 
On the other hand, adiponectin is a molecule that acts exactly in contrast to the mechanism of TNF- $\alpha$ and IL-6. Elevated levels of serum adiponectin correlate with lower risk of myocardial infarction in males. ${ }^{13}$ There is a theory suggesting that visceral obesity may result in lower levels of serum adiponectin, since serum adiponectin is positively correlated with weight reduction and negatively correlated with BMI and waist-to-hip ratio. Adiponectin increases insulin sensitivity and reduces TNF- $\alpha$ production. Systemic and local levels of TNF- $\alpha$ are associated with both psoriasis and obesity. Systemic inflammation of both conditions may lead to impaired glucose tolerance, endothelial dysfunction, and dyslipidemia, therefore increasing the cardiovascular risk of the patients.

A retrospective analysis of patients with immunemediated inflammatory disorders showed that patients with psoriasis had an elevated odds ratio of having cardiovascular risk factors such as atherosclerosis, hypertension, and type 2 diabetes. A small German cohort study revealed an increased prevalence and severity of coronary artery calcification in patients with psoriasis compared to non-psoriatic patients. ${ }^{14}$ An analysis of the British General Practice Research Database showed that patients with severe psoriasis had a higher relative risk of myocardial infarction that varied with age. The younger psoriasis patients showed the greatest risk. ${ }^{9}$ This was the first time that psoriasis was shown to be a risk factor for cardiovascular mortality independent of the other cardiovascular risk factors.

An immunological relationship between psoriasis and adipose tissue seems to exist. Adipose tissue itself is considered to be an organ of immunity. Adipocytes have TLRs that allow an immediate response to foreign pathogens and release cytokines like IL- 6 and TNF- $\alpha .{ }^{15}$ Interestingly, this process is dependent on leptin. Leptin is an adipocytokine that participates in the regulation of body weight and both innate and acquired immunity. ${ }^{16,17}$ Leptin is a stimulator of T-cells, and leptin deficiency in mice reverses autoimmune pathophysiology. This suggests a potential link between adipose tissue and psoriatic inflammation.

On the other hand, it should be mentioned that patients with psoriasis often reduce or give up leisure-time activities because of their impaired condition. A recent study showed that almost half of the patients reduce or completely give up their leisure-time activities. Sports activities are completely given up by $30.2 \%$ and reduced by $23.7 \% .^{18}$ This has an extra impact on the quality of life of psoriasis patients, because since they reduce their sport activities, the risk of being overweight or obese, and consequently of developing metabolic syndrome, is increased. Finally the social and psychological impact of psoriasis, which evokes feelings of shame, anxiety, and stress, leads to bad nutritional and dietary practices, which are an additional risk factor for developing metabolic syndrome. ${ }^{19}$

\section{Discussion}

The correlation between psoriasis and obesity has been well documented in diverse studies and control populations. The question is whether there is also a causative relationship between the two disorders. Furthermore, it is still questionable whether there is a direct positive correlation between BMI of psoriasis patients and dyslipidemia, insulin resistance, and cardiovascular risk. The scientists still wonder if different psoriatic phenotypes display different risks for increased BMI (or intra-abdominal fat), ie, increased risk for obesity and cardiovascular comorbidities. As far as the latter is concerned, it would be extremely interesting to know whether there is a difference in cardiovascular risk among typical plaque, palmoplantar, or pustular psoriasis.

There are more issues that need to be clarified, for example, whether the effect of systemic therapies for psoriasis correlate positively with a reduction of BMI or cardiovascular risk. Systemic therapies with biologics decrease the levels of inflammatory and pro-inflammatory cytokines, such as CRP, IL-6, and IL-1, but do not really influence endothelial dysfunction or insulin sensitivity. Patients with metabolic syndrome showed a reduction of CRP and an increase of adiponectin levels, when treated with etanercept $50 \mathrm{mg}$ once weekly for 4 weeks. On the other hand, they showed no difference in insulin sensitivity and BMI or waist-to-hip ratio. ${ }^{20}$ Further, methotrexate has been proven to decrease cardiovascular risk in patients with psoriasis and rheumatoid arthritis, especially when it is followed by folate supplementation. ${ }^{21}$ Systemic therapies in psoriasis may reduce the inflammatory milieu of the disease, but it still remains to be clarified whether and to which point they affect the real values of lipid profile, hypertension, obesity, and the additional components of metabolic syndrome.

The opposite question also interests the experts. Due to pharmacokinetic alterations, obesity can importantly affect the therapeutic efficacy in patients with psoriasis. The adipose tissue influences the volume and the drug distribution; in that way it would probably not be wrong to customize the therapeutic doses according to the patient's BMI. Since a large number of psoriasis patients are overweight or obese, the fixed-dose basis on which most systemic therapies of psoriasis are administered results in an inadequate treatment of those patients. 
It is not only interesting to evaluate whether the improvement of psoriasis leads to an improvement of cardiovascular risk but also whether direct changes of style life like weight reduction, smoking alteration, and alcohol reduction, as well as amelioration of lipid profile, glucose levels, and blood pressure help to improve the clinical picture of psoriasis.

Moreover, biomarkers of psoriatic inflammation must be identified. The absolute values of cytokines like TNF- $\alpha$ and IL-6 should be investigated to establish whether they directly correlate with psoriasis severity, adiposity, and risk of myocardial infarction. In a male population, adiponectin is conversely correlated with cardiovascular risk. ${ }^{13}$ Could this be the case also for psoriasis patients? There are also wellknown cytokines in psoriasis like CRP, and it would be very useful to follow their changes during the disease course.

The relation between psoriatic arthritis and obesity and metabolic syndrome should also be well documented. It is now known that psoriatic arthritis involves $10 \%-40 \%$ of psoriatic patients. ${ }^{21-24}$ Psoriatic arthritis itself constitutes a strong inflammatory situation of the human body. Is arthritis in psoriasis patients correlated with a higher risk for cardiovascular mortality? A genetic analysis should also be performed to evaluate the mutations in genes that regulate psoriasis, adiposity, and other components of the metabolic syndrome. In this way, there could possibly be a holistic approach to the disease which would lead to a better and deeper understanding of its pathophysiology.

\section{Disclosure}

The authors report no conflicts of interest in this work.

\section{References}

1. Henseler T, Christophers E. Disease concomitance in psoriasis. $J$ Am Acad Dermatol. 1995;32(6):982-986.

2. Krueger GG, Papp KA, Stough DB, et al. A randomized, double-blind, placebo controlled phase III study evaluating efficacy and tolerability of 2 courses of alefacept in patients with chronic plaque psoriasis. $J \mathrm{Am}$ Acad Dermatol. 2002;47(6):821-833.

3. Mease PJ, Goffe BS, Metz J, et al. Etanercept in the treatment of psoriatic arthritis and psoriasis: a randomized trial. Lancet. 2000;356(9227): 385-390.

4. Shirai K. Obesity as the core of the metabolic syndrome and the management of coronary heart disease. Curr Med Res Opin. 2004;20(3): 295-304.
5. Naldi L, Chatenoud L, Linder D, et al. Cigarette smoking, body mass index, and stressful life events as risk factors for psoriasis: results from an Italian case-control study. $J$ Invest Dermatol. 2005;125(1): 61-67.

6. Neimann AL, Shin DB, Wang X, et al. Prevalence of cardiovascular risk factors in patients with psoriasis. JAm Acad Dermatol. 2006;55(5): 829-835.

7. Herron MD, Hinckley B, Hoffman MS, et al. Impact of obesity and smoking on psoriasis presentation and management. Arch Dermatol. 2005;141(12):1527-1534.

8. Eckel RH, Grundy SM, Zimmet PZ. The metabolic syndrome. Lancet. 2005;365(9468):1415-1428.

9. Gelfand JM, Neimann AL, Shin DB, et al. Risk of myocardial infarction in patients with psoriasis. JAMA. 2006;296(14):1735-1741.

10. Sommer DM, Jenisch S, Suchan M, et al. Increased prevalence of the metabolic syndrome in patients with moderate to severe psoriasis. Arch Dermatol Res. 2006;298(7):321-328.

11. Sattar N, McCarey DW, Capell H, McInnes IB. Explaining how 'highgrade' systemic inflammation accelerates vascular risk in rheumatoid arthritis. Circulation. 2003;108(24):2957-2963.

12. Ronti T, Lupattelli G, Mannarino E. The endocrine function of adipose tissue: an update. Clin Endocrinol (Oxf). 2006;64(4):355-265.

13. Pischon T, Girman CJ, Hotamisligil GS et al. Plasma adiponectin levels and risk of myocardial infarction in men. JAMA. 2004;291(14): 1730-1737.

14. Ludwig RJ, Herzog C, Rostock A, et al. Psoriasis: a possible risk factor for development of coronary artery calcification. Br J Dermatol. 2007;156(2):271-276.

15. Coppack SW. Pro-inflammatory cytokines and adipose tissue. Proc Nutr Soc. 2001;60(3):349-356.

16. Pietsch J, Batra A, Stroh T, et al. Toll-like receptor expression and response to specific stimulation in adipocytes and preadipocytes. Ann NY Acad Sci. 2006;1072:407-409.

17. Rajala MW, Scherer PE. Mini review: the adipocyte - at the crossroads of energy homeostasis, inflammation and atherosclerosis. Endocrinology. 2003;144(9):3765-3773.

18. Leino M, Mustonen A, Mattila K, Koulu L, Tuominen R. Perceived impact of psoriasis on leisure-time activities. Eur J Dermatol. 2014; 24(2):224-228.

19. Feldman SR, Malakouti M, Koo JY. Social impact of the burden of psoriasis: effects on patients and practice. Dermatol Online J. 2014;20(8). pii: 13030/qt48r4w8h2.

20. Bernstein LE, Berry J, Kim S et al. Effects of etanercept in patients with the metabolic syndrome. Arch Intern Med. 2006;166(8):902-908.

21. Prodanovich S, Ma F, Taylor JR, et al. Methotrexate reduces incidence of vascular diseases in veterans with psoriasis or rheumatoid arthritis. J Am Acad Dermatol. 2005;52(2):262-267.

22. Grundy SM, Cleeman JI, Daniels SR, et al. Diagnosis and management of the metabolic syndrome: an American Heart Association/National Heart, Lung, and Blood Institute Scientific Statement. Circulation. 2005;112(17):2735-3752.

23. Eckel RH, Alberti KG, Grundy SM, Zimmet PZ. The metabolic syndrome. Lancet. 2010;375(9710):181-183.

24. Rocha-Pereira P, Santos-Silva A, Rebelo I, et al. Dyslipidemia and oxidative stress in mild and in severe psoriasis as a risk for cardiovascular disease. Clin Chim Acta. 2001;303(1-2):33-39.
Psoriasis: Targets and Therapy

\section{Publish your work in this journal}

Psoriasis: Targets and Therapy is international, peer-reviewed, open access journal focusing on psoriasis, nail psoriasis, psoriatic arthritis and related conditions, identification of therapeutic targets and the optimal use of integrated treatment interventions to achieve improved outcomes and quality of life. The manuscript management system

\section{Dovepress}

is completely online and includes a very quick and fair peer-review system. Visit http://www.dovepress.com/testimonials.php to read real quotes from published authors. 